\title{
Hepatic arterial chemotherapy for primary and metastatic liver cancers
}

\author{
William Ensminger \\ Upjohn Center for Clinical Pharmacology, Departments of Medicine and Pharmacology, University of Michigan Medical School, \\ Ann Arbor, Michigan 48109, USA
}

\begin{abstract}
Summary. Hepatic arterial chemotherapy represents a means of selectively exposing hepatic tumor to cytotoxic agents. Although 5-fluoro-2'-deoxyuridine has been shown to generate a higher response rate in the treatment of colorectal liver metastases than that achieved by intravenous infusion, responses are largely incomplete and rarely of long duration. This review describes the rationale for the use of the thymidine analogs 5-bromo-2'-deoxyuridine and 5-iodo-2'-deoxyuridine in hepatic arterial infusions and indicates how combination therapy adding radiotherapy, specifically with hepatic arterially administered yttrium-90 microspheres, might generate a new, more efficient and effective therapeutic approach.
\end{abstract}

\section{Rationale}

Cancer frequently grows within the liver and such growth is often a major source of morbidity and mortality. Although surgical resection can cure about one-third of patients having a solitary tumor nodule in the liver, it appears that less than $5 \%$ of patients with hepatic cancers fall into this category where resection can be attempted [2, 3, $13,15,29,30]$. Although the greatest benefit of other regional approaches aimed at the eradication of hepatic tumors would be anticipated to accrue in the small group of patients who truly have tumor confined to liver (but who are unresectable because of technical considerations), significant palliation might be possible and represent a worthwhile goal in the large number of patients with extensive tumor within the liver. In addition, systemic therapies might have more impact when applied to microscopic extrahepatic disease in this last group of patients.

Accordingly, there has been a great deal of interest in the treatment of hepatic cancer within the oncology community. Although hepatic arterial chemotherapy had been practiced previously in a limited number of centers, the development of a more convenient method for hepatic arterial therapy utilizing an implantable drug delivery system has led to the implantation of approximately 20000 of such systems at more than 1400 'centers' over the past 5 years. It is clear that recent interest in hepatic arterial chemotherapy as a treatment for hepatic cancer has been 'de-

Offprint requests to: W. Ensminger vice-driven' and not 'drug-driven' inasmuch as the agent used in essentially all hepatic arterial infusions has been 5-fluoro-2'-deoxyurdine (FdUrd), a drug that has been available for over 20 years. Recently conducted randomized studies from Sloan Kettering and the Northern California Oncology Group indicate that FdUrd, given by the hepatic arterial route, generates a higher objective response rate than an equitoxic dose of intravenous FdUrd given on the same two-weeks-out-of-four continuous infusion schedule $[19,23]$. Unfortunately, as a single agent, hepatic arterial FdUrd alone fails to induce tumor regression in about half of the patients treated, and the half that show an objective response frequently fail within the liver after 6-8 months of treatment [32].

Thus, devices (drug-delivery systems) now exist making protracted (hepatic arterial) drug infusions easier and more reliable. This technical capability, the demonstrated interest in hepatic arterial therapy as a treatment approach, the shortcomings of FdUrd, and the limitations imposed by a lack of other agents can be seen as factors driving the development of new and potentially more effective drug therapies using the hepatic arterial route of administration. It is evident that additional clinical programs are needed for hepatic arterial therapy.

A major rationale for hepatic arterial therapy relates to the importance of the hepatic artery in providing the blood supply to tumors growing within the liver. Recent elegant studies from the group at Sloan Kettering have confirmed the predominence of the hepatic artery as the source of nutrient blood flow to hepatic tumors [36] and that FdUrd uptake into human liver tumors is more than tenfold greater with hepatic arterial versus portal venous administration [38].

Pharmacokinetic theory predicts that it should be possible to generate higher and more selective drug exposure for intrahepatic tumors (fed by the hepatic artery) by direct hepatic arterial infusion of agents that have appropriate pharmacokinetic properties $[4,6]$. In particular, drugs which have a high total body clearance and a high hepatic extraction are most appropriate pharmacokinetically. Appropriately administered, hepatic arterial chemotherapy may generate higher levels of drug exposure for liver tumors than systemic tolerance would allow by intravenous administration, thereby allowing extension of the dose-response curve for antitumor activity. As illustrated in Fig. 1, hepatic tumor exposure may be high with concurrent low and tolerable systemic exposure with hepatic arterial infu- 


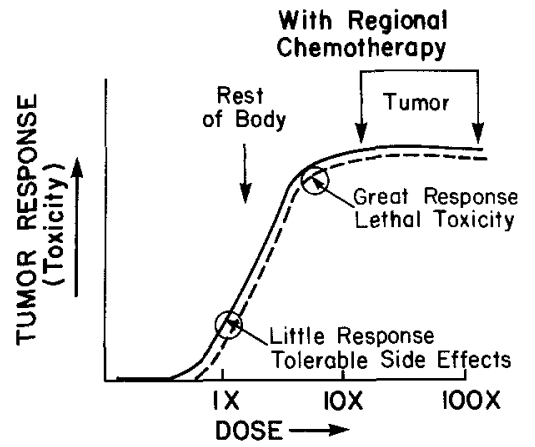

Fig. 1. Hypothetical dose-response curve with normal tissue (and tumor (-- - nearly superimposed. With intravenous therapy (O), tumor and normal tissue see same dose. With regional chemotherapy (uncircled arrows), regional tumor exposure is $10-100 \times$ exposure to rest of body

sion. With intravenous infusion at levels as high as those achieved in the liver with hepatic infusion, lethal toxicity may result as systemic dose-limiting tissues get too high an exposure.

As noted previously, hepatic arterial chemotherapy has played an important role in the development of drug-delivery systems for safe and reliable protracted, continuous infusion therapy. For drugs such as the nucleic acid precursor analog FdUrd, protracted exposure plays a crucial role in determining activity. At the cellular level, exposure during $\mathrm{S}$ phase is necessary inasmuch as DNA synthesis is a major target for such antimetabolites. Thus, extracellular drug levels must be maintained for numerous cell cycles to produce maximum effect in non-synchronized cells in culture (in vitro) or in tissues (in vivo). From a pharmacokinetic point of view, the high clearance and short $t_{t / 2}$ of many nucleic acid analogs necessitate constant infusion in vivo to maintain plasma (and extracellular) drug levels.

Hepatic arterial chemotherapy thus represents a method both for extending drug exposure to higher levels and for maintaining controlled drug levels for prolonged, defined time periods in vivo. As such, hepatic arterial chemotherapy represents an unique opportunity to extend studies on the selective effects of controlled drug exposure in cell cultures and animal models to patients with hepatic cancers. The relatively high tolerance of the liver to chemotherapeutic drugs, the minimal DNA synthesis in the liver and, the ability to monitor toxic and therapeutic effects readily within in the liver contribute to the rationale sub-

Table 1. Relevant factors favoring the use of 5-bromo-2'-deoxyuridine/5-iodo-2'-deoxyuridine

1. Potent cytotoxic agents (and radiosensitizers)

2. Crucial target effect to DNA thymine replacement by the analog

3. DNA incorporation a function of

(a) DNA synthetic rates

(b) Exposure (concentration, time)

4. Pharmacokinetic properties should allow increased exposure with regional intra-arterial administration

5. Selective inhibition of competing endogenous thymidylate synthesis may increase DNA incorporation of analog stantiating the use of hepatic arterial chemotherapy with DNA synthesis antimetabolites as a means to extend potentially meaningful in vitro observations into the clinical setting.

\section{5-Bromo-2'-deoxyuridine (BrdUrd) and \\ 5-iodo-2'-deoxyuridine (IdUrd) as new rational agents for hepatic arterial infusion}

As described below and noted in Table 1, there is evidence that the thymidine analogs, BrdUrd and IdUrd, represent rational agents to study in hepatic arterial therapeutics. Although these agents have existed for a long time, they have not previously been tested in hepatic arterial infusion. Recent pharmacokinetic data on these agents given intravenously has shown that both of these drugs have pharmacokinetic parameters (see below) making them reasonable to investigate in regional infusions $[27,37]$. In addition, although BrdUrd and IdUrd are directly cytotoxic to proliferating cells, it is likely that the excellent radiosensitizing properties they possess (upon incorporation into DNA) [11] will lead to continued expanded interest in their regional administration. Hepatic arterial chemotherapy with these agents, having incorporation into cellular DNA as a crucial target effect, represents an excellent opportunity to see whether in vitro/in vivo correlations are possible and whether they will facilitate application of biochemical pharmacology to clinical experimental therapeutics. In the long term, it appears probable that programs rationally designed would give rise to new, more effective regimens demonstrated by higher complete response rates and shorter treatments.

There are many variables that are probably extremely important to the design of therapies with BrdUrd and IdUrd having the best chance of success (Table 2). Although BrdUrd and IdUrd are both thymidine analogs, in which the 5-methyl group on the pyrimidine ring is replaced by bromine and iodine respectively, there is considerable evidence demonstrating that BrdUrd and IdUrd behave differently in biological systems. Stereochemical considerations would suggest that BrdUrd is the better thymidine analog since the van der Waals radius of bromine $(0.195 \mathrm{~nm})$ is closer to that of the methyl group $(0.20 \mathrm{~nm})$ of thymine than is the radius of iodine $(0.215 \mathrm{~nm})$. Consistent with this premise is evidence that for molar equivalent analog exposure, the percentage thymine replacement (in one human cell line) was greater for BrdUrd (18\%) than for IdUrd (9\%) [12]. On the other hand, per given level of thymine replacement, IdUrd generates considerably more cytotoxicity and X-ray sensitization in cultured cells [7, 12]. In cultured cells, BrdUrd incorporation leads to more ultraviolet sensitization than does IdUrd. In mouse tongue epithelial cells in vitro, neither BrdUrd nor IdUrd is stably incorporated into DNA and turnover times differ (1.0 days

Table 2. Important variables in the study of halogenated thymidine analogs

BrdUrd versus IdUrd

Dose rate

Schedule (duration)

Route administered (i.a. versus i.v.)

Modulation (temperature-sensitive inhibitors) 
for BrdUrd, 3.8 days for IdUrd [21]. Such differences, if extrapolated to a clinical setting, would appear relevant to the choice and schedule of analog to be used primarily for cytotoxic or radiosensitizing effect.

Recent clinical studies at the National Cancer Institute indicate that BrdUrd and IdUrd display several clinically relevant differences when administered intravenously to patients $[25-27,37]$. The total body clearance of BrdUrd was found to be $1.5-2.31 \mathrm{~min}^{-1} \mathrm{~m}^{-2}$ and the maximum tolerated dose rate and resultant plasma level for a 14-day i.v. infusion were $600-800 \mathrm{mg} \mathrm{m}^{-2}$ day $^{-1}$ and $1 \mu \mathrm{M}$ respectively. For IdUrd the total body clearance was found to be $0.751 \mathrm{~min}^{-1} \mathrm{~m}^{-2}$, and the corresponding maximum tolerated dose rate and plasma level (14-day i.v. infusion) were $1000 \mathrm{mg} \mathrm{m}^{-2} \mathrm{day}^{-1}$ and $5-6 \mu \mathrm{M}$. These preliminary results from the NCI indicate that the human body metabolizes BrdUrd more efficiently with a higher clearance than seen with IdUrd. Thus, on the basis of pharmacokinetic considerations, BrdUrd, given in an arterial infusion, should generate a twofold to threefold higher regional advantage than would a similar IdUrd infusion. On the other hand, a higher systemic concentration of IdUrd is tolerated in a 14-day intravenous infusion - which data indicate that the actual exposure advantage of a BrdUrd regional infusion over an IdUrd infusion (at maximum tolerated dose rates) would be less than anticipated.

One approach to gaining control of the many variables relevant to BrdUrd and IdUrd is to develop preclinical models and to validate them as to their predictability for the clinic. For example, preliminary data (Table 3 ), which we have obtained, demonstrate that rabbits [28] and dogs may provide pharmacokinetic information predictive of results in humans. Clearly, in all three species hepatic arterial BrdUrd achieves a significant (albeit a calculated estimate) regional advantage as compared to intravenous infusion.

We have applied an animal tumor model, the intrahepatically implanted VX2 tumor in rabbits, to the examination of the dependence of DNA incorporation on the sustained plasma drug level. In this system, the crucial target effect, incorporation of BrdUrd into DNA, follows saturating, non-linear kinetics as defined by a Longmuir type equation

$$
I=\frac{I_{\max } c}{c_{50}+c}
$$

where $I=$ percentage incorporation of BrdUrd into DNA; $I_{\max }=$ maxium percentage incorporation of BrdUrd; $c=$ steady-state plasma concentration of BrdUrd; $c_{50}=$ plasma BrdUrd concentratzion at half-maximal incorporation. The curve plotting incorporation into bone marrow always lies above that for tumor (Fig. 2) as ascertained in 24-h intravenous infusions with measurements of steady-state sys-

Table 3. BrdU pharmacokinetics in rabbits, dogs and humans

\begin{tabular}{llllr}
\hline Subjects & $\begin{array}{l}\mathrm{CL}_{\mathrm{TB}} \\
(\mathrm{ml} / \mathrm{min})\end{array}$ & $\begin{array}{l}\text { Normalized } \\
\left(\mathrm{ml} / \mathrm{kg}^{-1} \mathrm{~min}^{-1}\right)\end{array}$ & $\begin{array}{l}\text { Hepatic } \\
\text { extraction } \\
\text { ratio }\end{array}$ & $\begin{array}{l}\text { Hepatic } \\
\text { regional } \\
\text { advantage }\end{array}$ \\
\hline Rabbits & $180-220$ & $45-55$ & $0.66-0.75$ & $30-50$ \\
Dogs & $1000-1600$ & $33-50$ & $0.90-0.95$ & $\begin{array}{r}100-350 \\
40-100\end{array}$ \\
Humans & $2500-3500$ & $35-50$ & $0.75-0.90$ & 40.00 \\
\hline
\end{tabular}

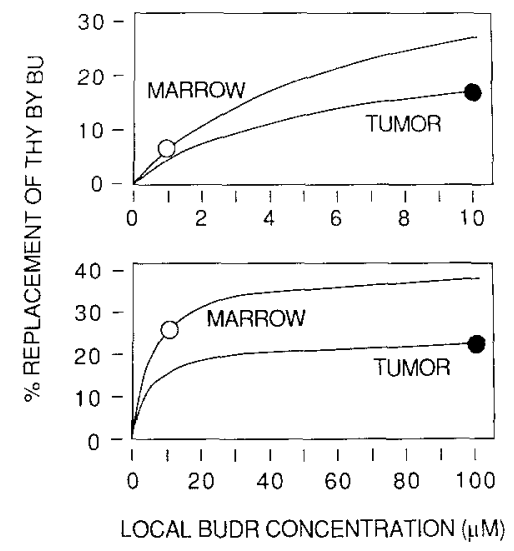

Fig. 2. Predicted incorporation of BrdUrd (BU or BUDR) into DNA (thymine replacement of VX2 tumor and of bone marrow) assuming tenfold regional exposure advantage such as might be achieved through hepatic arterial infusion. Top curves, low dose rate; bottom high dose rate. Marrow $(O)$ and tumor $(\mathbf{)}$ incorporation falls in each case on pharmacodynamically determined curves for marrow and tumor, respectively

temic arterial plasma levels [39]. These tissue-dependent incorporation profiles give rise to an important prediction. Although pharmacokinetic parameters may allow a selective increase in hepatic tumor exposure with hepatic arterial infusion, the impact on what actually is most important, i. e. DNA incorporation, will be less selective at high than at low dose rates. If, for example, one assumes the achievement of a tenfold regional advantage, at low dose rates (top, Fig. 2), tumor incorporation (filled circle) is greater than marrow incorporation (open circle). At high dose rates (bottom, Fig. 2), despite higher local BrdUrd levels, tumor incorporation (filled circle) is less than marrow (open circle). Thus, more is not always better! Pilot data in the VX2 system have indicated that the predication is correct, showing a decline in selective uptake as the hepatic arterial dose rate increases even from $10 \mathrm{mg} \mathrm{kg}^{-1}$ day $^{-1}$ to $20 \mathrm{mg} \mathrm{kg}^{-1}$ day $^{-1}$.

In cultured cells, inhibition of thymidylate synthase reduces endogenous production of thymidine monophosphate and results in increased salvage of exogenous thymidine or of exogenously provided thymidine analogs. Incorporation of the thymidine analog BrdUrd can be increased as much as tenfold in cultured cells when FdUrd is used to inhibit thymidylate synthase via FdUMP formation [9]. Recognizing the limitations of intravenous replacement of DNA thymine residues by iodouracil residues, the intramural group at the NCI has initiated a phase I clinical study of combined intravenous infusion of IdUrd with FdUrd. At the University of Wisconsin, a phase I protocol, involving hepatic arterial infusion of IdUrd plus fluorouracil is underway as well [40].

Inasmuch as FdUrd and 5-fluorouracil both have pharmacokinetic properties leading to an improved regional exposure advantage with hepatic arterial infusion, combination of FdUrd or fluorouracil with BrdUrd or IdUrd in hepatic arterial infusions would appear reasonable. Using the rabbit VX2 system, we have found that concurrent administration of FdUrd and fluorouracil at clinically relevant dose rates does not appreciably change BrdUrd incorporation into replicating marrow cells but does increase (selectively) incorporation into VX2 some twofold. 
After additional studies of dose range in cell culture and in the VX2 system, clinical studies will be initiated using a gas chromatography/mass spectrometry assay of incorporated BrdUrd combined with the application of two stable isotopes of BrdUrd to determine whether FdUrd or fluorouracil can selectively increase BrdUrd incorporation into hepatic tumors versus normal tissues.

\section{Radiation \pm hepatic arterial chemotherapy}

Radiation, as a sole modality, has been of modest benefit in the treatment of hepatocellular carcinoma. This is chiefly because the tolerance dose to the whole liver for radiation generally is accepted to be approximately $3500 \mathrm{cGy}$. This conclusion is derived from retrospective reviews, chiefly of patients from Memorial Hospital [22] and Stanford University [34]. These patients were treated once daily, typically with one field a day. If the data from these two series are pooled, four out 48 patients treated with less than 3500 cGy developed radiation hepatitis: three at $3500 \mathrm{cGy}$ and one at $3000 \mathrm{cGy}$. None of these cases was either persistent or fatal. Seven of the 24 patients treated with 3500-4000 cGy developed radiation hepatitis, which in some cases was persistent or fatal.

Given these restrictions on the dose of radiation that can be safely administered, it can be appreciated why there has been little enthusiasm for radiation as a sole modality. In one series, 31 patients who were unresectable were treated with between $1000 \mathrm{cGy}$ and $3600 \mathrm{cGy}$, with only one survivor past 1 year [8]. Similar results were obtained in a small randomized trial between combination chemotherapy and radiation therapy (3000cGy in 200-cGy fractions) followed 2 months later by chemotherapy. Six out of eight patients progressed either during radiation or after radiation, but prior to chemotherapy [5].

Because of discouraging results using surgery, radiation, and chemotherapy as single modalities, combination approaches have been attempted. Simultaneous intra-arterial hepatic chemotherapy (bolus doxorubicin and continuous infusion of fluorouracil) and radiation (1500-2400 cGy in 300-cGy fractions) has been investigated in 13 patients by Friedman et al. [14]. Six of the 13 patients (46\%) had an objective response in this pilot study without significant toxicity, suggesting that this general approach may have some merit. Order and colleagues have used a combination of external-beam irradiation, radiolabeled anti-ferritin monoclonal antibodies, chemotherapy, and surgery with encouraging results [33], although it has been noted that the complexity of this regimen makes it difficult to determine the relative contributions of each form of treatment [31].

In an attempt to improve upon the results of intra-arterial chemotherapy alone for cancers metastatic to liver, some investigators have used combined intra-arterial chemotherapy and radiation for treatment (for review see [24]). Typically, the liver has been radiated to a total dose of 2500-3000 cGy with a single fraction of $200-300 \mathrm{cGy}$ given per day. Chemotherapy (usually fluorouracil or FdUrd) has been given concurrently. The reported response rates have been in the range of $48-70 \%$, but rigid criteria for judging a response usually are lacking. Overall, this treatment has been well tolerated. A recent study reports use of a schedule of hyperfractionated radiation, using $150 \mathrm{cGy}$ twice a day to a total dose of $2100 \mathrm{cGy}$ [35].
This was combined with hepatic arterial FdUrd in four patients and intravenous fluorouracil in eight patients. Interpretation of this study is difficult owing to the small number of patients and a lack of objective response criteria. However, two patients were alive without evidence of disease at 24 months and toxicity was minimal. This study suggests that this may be a useful approach.

\section{Yttrium-90 microspheres and tumor hypervascularity}

As tumors grow, the evoked new capillary bed develops at the periphery, so that the most vascularized area is the outer shell of the nodule [41]. Although the central core of many tumor nodules in the liver is hypovascular, the periphery of the tumor nodule is generally hypervascular relative to normal liver, as demonstrated by nuclear tomographic scans after hepatic-arterial injection of TcMAA $[16,17]$. The microvascular pattern is consistent with the distribution of tumor cell viability and growth, the central core of the tumor often being necrotic while the peripheral rim of actively proliferating tumor cells has an excellent blood supply [1, 41]. The density of vessels in the hypervascular regions of tumor nodules appears to be twofold to sixfold greater than in normal liver [10]. These observations suggest that relative tumor hypervascularity might be used for selective therapeutic advantage with hepatic arterial injection of therapeutic microspheres.

Microspheres of $20-80 \mu \mathrm{m}$ diameter, when injected as a homogeneous suspension into the hepatic artery, should lodge in the hepatic arterial microvasculature in direct proportion to regional blood flow throughout that watershed. As mentioned earlier, the hepatic-arterial injection of TcMAA (lung scanning agent) with nuclear tomography provides a means to determine the relative blood flow distribution between normal liver and tumor nodules within liver and, thus, to monitor selective delivery of therapeutic microspheres to tumor [16].

A 22- $\mu \mathrm{m}$ glass microsphere (TheraSphere) has been developed in which yttrium-89 oxide is incorporated into the glass matrix and is activated by neutron bombardment to form the beta-emitting isotope yttrium- $90\left({ }^{90} \mathrm{Y}\right)$ prior to use of the spheres as radiotherapeutic vehicles [42]. In dogs to which arterial ${ }^{90} \mathrm{Y}$ microspheres were administered at progressively higher dosages, radiation exposures in excess of $30000 \mathrm{rad}$ did not cause total hepatic necrosis and were compatible with survival [42]. No microspheres distributed to the bone marrow and absolutely no myelosuppression were encountered in any animal. Using a protocol supported by the dog studies, patients with hepatic tumors have received hepatic arterially administered TheraSpheres. No significant toxicity has been demonstrated for absorbed doses between 5000 and 10000 rad to the liver and up to $20000 \mathrm{rad}$ to the tumor (owing to tumor hypervascularity) $[18,20]$. After dose-seeking phase I studies are complete, antitumor activity will be assessed at the maximum tolerated dose (which appears to exceed $10000 \mathrm{rad}$ ).

\section{Conclusion}

A reasonable therapeutic aim is the development of new regimens for the treatment of primary and metastatic liver cancers that can efficiently (in 1-2 months of treatment) and effectively (complete response rate $>80 \%$ ) achieve eradication of surgically unresectable cancer. In this review, the potential for the development of new regimens 
utilizing BrdUrd and IdUrd has been described. Combination of these potent radiosensitizers with ${ }^{90} \mathrm{Y}$ microspheres (both administered via the hepatic artery) may generate markedly improved selectivity and would represent a potent new approach to treatment. Toxicity studies in dogs indicate that such will be feasible without untoward hepatic toxicity [43]. Should such treatment prove effective in the clinic, a few patients with surgically unresectable hepatic cancer confined to the liver might be cured and many more with bulk hepatic tumor might achieve improved palliation.

Acknowledgements. The data described in this overview/review coming from the University of Michigan represent the considerable skilled efforts of my associates including: Phil Stetson, Ira Wollner, Jim Andrews, Suzette Walker-Andrews, Jim Knol, Conrad Knutsen, Diane Prieskorn, Patti Smith, Norma Johnson, and others. The superb assistance of my secretary, Bambi Castello is also appreciated.

\section{References}

1. Ackerman NB, Heckmer PA (1980) The blood supply of experimental liver metastases. V. Increased tumor perfusion with epinephrine. Am J Surg 140:625

2. Adson MA, Sheedy PF (1974) Resection of primary hepatic malignant lesions. Arch Surg 108: 599

3. Bengmark S, Borjesson B, Hafstrom L (1971) The natural history of primary carcinoma of the liver. Scand J Gastroenterol 6: 351

4. Chen HSG, Gross JF (1980) Intra-arterial infusion of anticancer drugs: Theoretic aspects of drug delivery and review of responses. Cancer Treat Rep 64: 31

5. Cochrane AMG, Murray-Lyon IM, Brinkley DM, Williams R (1977) Quadruple chemotherapy versus radiotherapy in treatment of primary hepatocellular carcinoma. Cancer 40:609

6. Collins JM (1984) Pharmacologic rationale for regional drug delivery. J Clin Oncol 2: 498

7. Djordjevic B, Szybalski W (1960) Genetics of human cell lines. III. Incorporation of 5-bromo- and 5-iododeoxyuridine into the deoxyribonucleic acid of human cells and its effect on radiation sensitivity. J Exp Med 112: 509

8. El-Domeiri AA, Huvos AG, Goldsmith HS, Foote FW Jr (1971) Primary malignant tumors of the liver. Cancer 27:7

9. Ellwart J, Dormer P (1985) Effect of 5-fluoro-2'-deoxyuridine (FdUrd) on 5-bromo-2'-deoxyuridine (BrUrd) incorporation into DNA measured with a monoclonal BrdUrd antibody and by the BrdUrd/Haechst quenching effect. Cytometry $6: 513$

10. Ensminger WD and Gyves JW (1984) Regional cancer chemotherapy. Cancer Treat Rep 68: 101

11. Erikson RL, Szybalski W (1961) Molecular radiobiology of human cell lines. I. Comparative sensitivity to X-rays and ultraviolet light of cells containing halogen-substituted DNA. Biochem Biophys Res Commun 4(4): 258

12. Erikson RL, Szybalski W (1963) Molecular radiobiology of human cell lines. V. Comparative radiosensitizing properties of 5-halodeoxycytidines and 5-halodeoxyuridines. Radiat Res 20: 252

13. Fortner JG, Dong KK, Maclean BJ, Barrett MK, Iwatsuki S, Turnbull AD, Howland WS, Beattie EJ Jr (1978) Major hepatic resection for neoplasia. Ann Surg 188: 363

14. Friedman MA, Volberding PA, Cassidy MJ, Resser KJ, Wasserman TH, Phillips TL (1979) Therapy for hepatocellular cancer with intrahepatic arterial adriamycin and 5-fluorouracil combined with whole-liver irradiation: A Northern California Oncology Group study. Cancer Treat Rep 63: 1885

15. Geddes EW, Falkson G (1970) Malignant hepatoma in the Bantu. Cancer 25: 1271
16. Gyves J, Ensminger W, Yang P, Thrall J, Cho K, Walker S (1982) Clinical utility of microspheres to assess and attack hepatic tumor microcirculation. Clin Res 30: 418 A

17. Gyves J, Ensminger W, Thrall J, Cho K, Walker S (1982) Dependence of hepatic tumor vascularity on tumor size. Clin Res 30:747 A

18. Herba MJ, Illescas FF, Thirwell M, Rosenthall L, Atri M, Bret PM (1987) Hepatic malignancy: improved delivery methods for treatment with intraarterial Y-90. Radiol 165 (P): 181

19. Hohn D, Stagg R, Friedman M, Ignoffo R, Rayner A, Hannigan J, Lewis B (1987) The NCOG randomized trial of intravenous (IV) vs hepatic arterial (IA) FUDR for colorectal cancer metastatic to the liver. Proc Am Soc Clin Oncol Annu Meet 6: 85

20. Houle S, Yip T-CK, Shepherd FA, Rotstein LE, Theis B, Cawthorn R, Barnes K (1987) Pilot trial of Y-90 glass micropheres in the treatment of primary hepatocellular carcinoma. Radiol $165(\mathrm{P}): 98$

21. Hume WJ, Saffhill R (1986) Iodo- and bromodeoxyuridine are excised at different rates from DNA of mouse tongue keratinocytes in vitro. Chem Biol Interact 60: 227

22. Ingold JA, Reed GB, Kaplan HS, Bagshaw MA (1965) Radiation hepatitis. Am J Roentgenol 93: 200

23. Kemeny N, Daly J, Reichman B, Geller N, Botet J, Oderman P (1987) Intrahepatic or systemic infusion of fluorodeoxyuridine in patients with liver metastases from colorectal carcinoma. Ann Int Med 107: 459

24. Kinsella TJ (1983) The role of radiation therapy alone and combined with infusion chemotherapy for treating liver metastases. Sem Oncol 10:215

25. Kinsella TJ, Russo A, Mitchell JB, Rowland J, Jenkins J, Schwade J, Myers CE, Collins JM, Speyer J, Kornblith P, Smith B, Kufta C, Glatstein E (1984) A phase I study of intermittent intravenous bromodeoxyuridine (BUDR) with conventional fractionated irradiation. Int $J$ Radiat Oncol Biol Phys 10: 69

26. Kinsella TJ, Russo A, Mitchell JB, Collins JM, Rowland J, Wright D, Glatstein E (1985) A phase I study of intravenous iododeoxyuridine as a clinical radiosensitizer. Int $\mathbf{J}$ Radiat Oncol Biol Phys 11: 1941

27. Klecker RW, Jenkins JF, Kinsella TJ, Fine RL, Strong JM, Collins JM (1985) Clinical pharmacology of 5-iodo-2'-deoxyuridine and 5-iodouracil and endogenous pyrimidine modulation. Clin Pharmacol Ther 38: 45

28. Knol JA, Stetson PL, Wagner J, Ensminger W (1987) Hepatic pharmacokinetics of 5-bromo-2'-deoxyuridine (BUDR) in the rabbit. Clin Pharmacol Ther 41(2): 171

29. Latham F Jr, Foster JH (1967) Hepatic resection for metastic cancer. Am J Surg 113: 551

30. Lee YT (1977) Systemic and regional treatment of primary carcinoma of the liver. Cancer Treat Rev 4: 195

31. Moertel CG (1986) The ${ }^{131}$ I antiferritin breakthrough. J Clin Oncol 4: 608

32. Niederhuber JE, Ensminger WD, Gyves JW, Thrall J, Walker S, Cozzi E (1984) Regional chemotherapy of colorectal cancer metastatic to the liver. Cancer 53: 1336

33. Order SE, Stillwagon GB, Klein JL, Leichner PK, Siegelman SS, Fishman EK, Ettinger DS, Haulk T, Kopher K, Finney K, Surdyke M, Self S, Leibel S (1985) Iodine-131 antiferritin, a new treatment modality in hepatoma. A radiation therapy Oncology Group Study. J Clin Oncol 3: 1573

34. Phillips R, Karnofsky DA, Hamilton LD, Nickson JJ (1954) Roentgen therapy of hepatic metastases. Am J Roentgenol 71: 826

35. Raju PI, Maruyama Y, DeSimone P, MacDonald J (1987) Treatment of liver metastases with a combination of chemotherapy and hyperfractionated external radiation therapy. Am J Clin Oncol 10(1): 41

36. Ridge JA, Bading JR, Gelbard AS, Benva RS, Daly JM (1987) Perfusion of colorectal hepatic metastases: relative distribution of flow from the hepatic artery and portal vein. Cancer 59: 1547 
37. Russo A, Gianni L, Kinsella TJ, Klecker RW, Jenkins J, Rowland J, Glatstein E, Mitchell JB, Collins J, Myers C (1984) Pharmacological evaluation of intravenous delivery of 5-bromodeoxyuridine to patients with brain tumors. Cancer Res 44: 1702

38. Sigurdson ER, Ridge JA, Kemeny N, Daly JM (1987) Tumor and liver drug uptake following hepatic artery and portal vein infusion. J Clin Oncol 5: 1836

39. Stetson PL, Johnson N, Knutsen C, Knol JA, Ensminger WD (1987) Pharmacodynamics of 5-bromo-2'-deoxyuridine (BUDR) in the rabbit hepatic VX2 tumor model. Clin Pharmacol Ther 41(2): 224

40. Trump D, Benson A, Weese J, Fischer P, Wilson J, Ramirez G, Grem J, Simon K, Alberti D, Tutsch K (1986) Phase I trial of hepatic artery infusion (HAI) of 5-iodo-2'-deoxyuridine (IdUrd) and 5-fluorouracil (5FU) in patients with advanced hepatic malignancy: biochemically based combination therapy. Proc Am Assoc Cancer Res Annu Meet 27: 295

41. Warren BA (1979) The vascular morphology of tumors. In: Petersen HI (ed) tumor blood circulation: angiogenesis, vascular morphology and blood flow of experimental and human tumors. CRC Press Inc., Florida, pp 1-47

42. Wollner IS, Knutsen C, Smith P, Prieskorn D, Chrisp C, Andrews J, Juni J, Warber S, Klevering J, Crudup J, Ensminger W (1988) Effects of hepatic arterial yttrium-90 glass microspheres in dogs. Cancer (in the press)

43. Wollner IS, Knutsen CA, Ullrich KA, Chrisp CE, Juni JE, Andrews JC, Tuscan MJ, Stetson PL, Ensminger WD (1987) Effects of hepatic arterial yttrium-90 microsphere administration alone and combined with regional bromodeoxyuridine infusion in dogs. Cancer Res 47: 3285 